unclear. In England and Wales, 7.6\% infants are born before 37 weeks gestation; this is higher (16.2\%) among those of South Asian ethnicity. We examined PCHI risk by age 11 years in a prospective UK-wide cohort of children born before UNHS. We tested the hypothesis that lower gestational length is associated with higher PCHI risk after adjusting for NICU/ SCBU admission and ethnicity.

Methods PCHI risk (cumulative incidence) was based on parental report of hearing impairment and associated treatment at ages nine months, three, five, seven and 11 years for 19518 children participating in the Millennium Cohort Study. The association of PCHI (defined by provision of hearing aids/cochlear implants, persisting hearing impairment at final report, and absence of glue ear) with gestational length was investigated using multivariable discrete-time survival analysis, adjusting for NICU/SCBU admission, ethnicity and other confounding factors, and weighting for survey design (Stata: Release 14; StataCorp LP).

Results Parents reported no health problems in the first week after birth in 10247 (52.4\%), children, neonatal illness without NICU/SCBU admission in 6781 (38.0\%), and NICU/SCBU admission in 1785 (9.6\%). PCHI was ascertained in 44 children by age 11 years. PCHI risk was 1.0 per 1000 children (95\% CI: $0.6-1.6)$ by age 9 months, rising by age five to 1.5 $(1.0-2.2)$ and by age 11 to $2.1(1.5-3.0)$. PCHI risk by age 11 was not associated with gestational length (hazard ratio (HR): 1.00, 95\% CI: 0.98-1.03), but was associated with parental report of neonatal illness with or without NICU/ SCBU admission (HR: 6.33; 2.27-17.63 and 2.62; 1.15-5.97, respectively) and Bangladeshi or Pakistani ethnicity (HR: 2.78; 1.06-7.31).

Conclusion In this cohort, born before UNHS, PCHI risk was highest in infancy. Neonatal illness, irrespective of NICU/ SCBU admission, and not gestational length, increased PCHI risk by age 11 years. Further research should explore the observed increased PCHI risk in children of Bangladeshi or Pakistani ethnicity, and the relevance for UNHS of PCHI with onset or diagnosis after infancy.

ESRC-funded PhD ES/J500185/1.

\section{P19 FATHER INVOLVEMENT AND ATTITUDES IN EARLY CHILD-REARING AND DEPRESSIVE SYMPTOMS IN THE PRE-ADOLESCENT PERIOD IN A UK BIRTH COHORT}

C Opondo*, M Redshaw, M Quigley. Nuffield Department of Population Health, University of Oxford, Oxford, UK

\subsection{6/jech-2017-SSMAbstracts. 121}

Background Although much of the research on the influence of parenting on child development has emphasised the mother's role, increasing evidence highlights the important role of fathers in the development, health and well-being of their children. In this study we aimed to investigate the association between father involvement in early child upbringing and depressive symptoms in the same children in their pre-teenage years.

Methods Data for the study came from the Avon Longitudinal Study of Parents and Children (ALSPAC) cohort from the southwest of England. The outcome, depressive symptoms, was measured using the short Moods and Feelings Questionnaire (sMFQ) when the children were 9 and 11 years of age. The primary exposure was paternal involvement measured using scores derived from factor analysis of fathers' report of their participation in, understanding of, and feelings about their child's early upbringing.

Results Three factors were identified in the factor analysis. Scores on factor 1 measured fathers' emotional response to the child; scores on factor 2 measured the frequency of fathers' involvement in domestic and childcare activities; scores on factor 3 measured fathers' feelings of security in their role as parent and partner. Children of fathers with high scores on factors 1 and 3 had 12\% (OR 0.88, 95\% CI 0.81-0.97, $\mathrm{p}=0.009$ ) and $9 \%$ (OR 0.91, 95\% CI 0.82-1.00, $\mathrm{p}=0.040$ ) respectively lower adjusted odds of reporting more depressive symptoms at 9 years. There was no evidence of a difference in depressive symptoms associated with factor 1 and factor 3 scores at 11 years. However, there was weak evidence of a $13 \%$ increase in odds of reporting more depressive symptoms associated with 1 unit increase in factor 2 scores at age 9 years (OR 1.13, 95\% CI 0.99-1.29, $\mathrm{p}=0.061$ ) and a $16 \%$ increase at 11 years (OR 1.16, 95\% CI 1.01-1.34, p=0.040). Conclusion Positive psychological and emotional aspects of father involvement in children's early upbringing, but not the quantity of direct involvement in childcare, may protect children against developing symptoms of depression in their preteen years.

\section{P20 CUMULATIVE EFFECT OF ADVERSE CHILDHOOD EXPERIENCES ON AFFECTIVE SYMPTOM TRAJECTORIES IN ADULTHOOD: EVIDENCE FROM A BRITISH BIRTH COHORT}

${ }^{1}$ EJ Thompson*, ${ }^{2} \mathrm{M}$ Richards, ${ }^{1} \mathrm{D}$ Gaysina. 'School of Psychology, University of Sussex, Brighton, UK; ${ }^{2}$ MRC Unit for Lifelong Health and Ageing, University College London, London, UK

\subsection{6/jech-2017-SSMAbstracts. 122}

Background Previous studies have shown that specific types of adverse childhood events (ACEs), such as parental divorce and parental psychopathology, pose a risk for the development of affective symptoms in adulthood (AS). However, a majority of this evidence is based on single types of retrospectively reported ACEs. This is problematic as ACEs tend to be interrelated and often co-occur.

Methods We used the data from the MRC National Survey of Health and Development (NSHD). This is an ongoing longitudinal study of 5362 women and men who were born in Britain in1946. Later life AS were measures using the General Health Questionnaire (GHQ) at ages 53 y, 60-64 y and 69.

Multiple imputation was implemented on each ACE predictor and a cumulative risk index was derived though summing the number of adversities experienced by each participant $(0$, $1,2,3 \ldots 20)$ before age $16 \mathrm{y}$. The effect of cumulative ACEs on AS at each time point $(53,60-64$ and 69) was examined using linear regression.

Results Preliminary analyses revealed a significant association was found between cumulative ACEs and AS at ages 60-64, $\beta(1,2183)=0.07, \quad p=0.002$, and $69, \quad \beta(1,2110)=0.07$, $\mathrm{p}=0.003$, but not age $53 \beta(1,2900)=0.04, \mathrm{p}=0.058$. Further to this growth mixture modelling will be used to model latent trajectories of AS between age 53 and 69 years and the effect of cumulative ACEs will be examined.

Discussion These findings will be presented in light of the growing evidence for the negative effects of ACEs on health and wellbeing in later life. Furthermore, we will discuss how 
this research informs prevention for the development of psychopathology across the life course.

\section{P21 PATTERNS OF BREASTFEEDING AT 3 MONTHS POSTPARTUM IN THE ALBERTA PREGNANCY OUTCOMES AND NUTRITION (APRON) STUDY}

${ }^{1} \mathrm{M}$ Jarman*, ${ }^{2} \mathrm{PJ}$ Robson, ${ }^{3} \mathrm{Y}$ Shen, ${ }^{1} \mathrm{M}$ Madsen, ${ }^{1} \mathrm{RC}$ Bell. ${ }^{1}$ Dept of Agriculture Food and Nutrition Science, University of Alberta, Edmonton, Canada; ${ }^{2}$ Cancer Measurement, Outcomes, Research and Evaluation, Cancer Control Alberta, Alberta Health Services, Edmonton Canada; ${ }^{3}$ School of Public Health, University of Alberta, Edmonton, Canada

\subsection{6/jech-2017-SSMAbstracts.123}

Background Infant feeding is often characterised in broad categories, such as exclusive breastfeeding, partial breastfeeding and formula feeding. However there is little information about detailed patterns of feeding within these groups. The purpose of this study was to examine patterns of breastfeeding from 3 day prospective feeding diaries to describe how women feed their babies.

Methods The APrON study is a prospective study of women during pregnancy and their children. At 3 months postpartum women completed a prospective breastfeeding diary which collected information on the number of feeds, and duration and method of every feed over 3 days. A total of 1080 women completed the breastfeeding diaries.

Results Women reported feeding their babies using combinations of up to 4 different methods (at breast, expressed breastmilk in a bottle, formula, mixed breastmilk and formula in the same bottle) in any one day. For the ongoing analyses women were categorised into 4 groups based on the number and type of feeds reported/day: 1) at breast only $(n=622), 2)$ at breast and expressed breastmilk $(n=223), 3)$ mixed feeding using 2 methods $(n=123)$ and, 4) mixed feeding using 3 or 4 methods $(n=112)$. The proportion of total feeds at breast was $80 \%, 75 \%$ and $64 \%$ for the mothers in the 'at breast and expressed milk', 'mixed feeding using 2 methods' and 'mixed feeding of 3 using 4 methods' groups, respectively. In turn the average daily duration of at breast feeds was longer in women who fed at breast only compared to those in any other method group. Women who fed 'at breast and expressed milk', 'mixed feeding of 2 methods' and 'mixed feeding of 3 or 4 methods' fed, on average, for $18 \mathrm{~min}$, $17 \mathrm{~min}$ and $27 \mathrm{~min}$ less at breast per day, respectively, compared to women who fed at breast only (all $\mathrm{p}=<0.01$ ). There were no differences in the average number of daily feeds between the 4 method groups.

Women use many different combinations of methods within a day to feed their 3 month old babies. Using fewer groups to classify women ignores the 'real life' complexity of feeding patterns. Our data showed that those who used a combination of methods to feed their babies fed had a lower a frequency and total duration of feeding at breast. This could be important to understanding variation in maternal energy expenditure and infant growth, development and health in the future.

In collaboration with the ENRICH team.

\section{P22 CHILDHOOD ADVERSITY, ADULT SOCIOECONOMIC STATUS AND RISK OF WORK DISABILITY - A PROSPECTIVE COHORT STUDY}

${ }^{1} \mathrm{Jl}$ Halonen* ${ }^{*}{ }^{1,2,3} \mathrm{M}$ Kivimäki, ${ }^{4,5} \mathrm{~J}$ Vahtera, ${ }^{3} \mathrm{~J}$ Pentti, ${ }^{1} \mathrm{M}$ Virtanen, ${ }^{1} \mathrm{~J}$ Ervasti, ${ }^{1} \mathrm{~T}$ Oksanen, ${ }^{1} \mathrm{~T}$ Lallukka. ${ }^{1}$ Work Disability Prevention, Finnish Institute of Occupational Health, Helsinki, Finland; '2Department of Epidemiology and Public Health, University College London, London, UK; ${ }^{3}$ Department of Public Health, University of Helsinki, Helsinki, Finland; ${ }^{4}$ Department of Public Health, University of Turku, Turku, Finland; ${ }^{5}$ Turku University Hospital, Turku, Finland

\subsection{6/jech-2017-SSMAbstracts. 124}

Background Employees' health is dependent on individual and social factors operating from across the life course. Studies have shown that negative life events during childhood or low socioeconomic status (SES) in adulthood may predict decreased labour market participation. However, the combined effects of childhood adversities and low SES in adulthood on work disability are not known.

Methods We included 34384 employed Finnish Public Sector study participants who responded to questions about childhood adversities (none versus any adversity, e.g., parental divorce or financial difficulties) in 2008, and whose adult SES (based on occupational status) in 2008 was available. We categorised exposure into four groups: neither (reference), childhood adversity only, low SES only, or both. Participants were followed from 2009 until the first period of register-based work disability (sickness absence $>9$ days or disability pension) due to any cause, musculoskeletal or mental disorders; retirement; death; or end of follow-up (December 2011). We ran cox proportional hazard models adjusted for behavioural, health- and work-related covariates.

Results When compared to those with neither exposure, hazard ratio (HR) for work disability from any cause was increased among participants with childhood adversity, with low SES, and those with both exposures. Low adult SES was a stronger predictor ( $\mathrm{HR} 2.38,95 \% \mathrm{CI}=2.14$ to 2.64 ) of disability due to musculoskeletal disorders than childhood adversity $(1.11,95 \% \mathrm{CI}=1.00$ to 1.23$)$. The difference between the exposures as predictors of disability due to mental disorders was smaller (adversity: $1.40,95 \% \mathrm{CI}=1.23$ to 1.59 ; low SES: $1.21,95 \% \mathrm{CI}=1.03$ to 1.42 ). The highest risk was observed in those with both exposures $(2.53,95 \% \mathrm{CI}=2.29$ to 2.79 for musculoskeletal disability).

Discussion Exposure to adversities in childhood and low SES in adulthood were associated with work disability. Exposure to both these risk factors was associated with the highest work disability risk, although this was additive rather than synergistic effect. Childhood adversity was associated with disability due to mental disorders in particular, whereas low adult SES was more strongly associated with disability due to musculoskeletal disorders.

\section{P23 POSSIBLE IMPLICATIONS OF A PERCEIVED DIVIDE BETWEEN LAY AND OFFICIAL KNOWLEDGE IN AN AREA OF COMPROMISED AND POLLUTED ENVIRONMENT, AND HIGH DEPRIVATION, IN NORTH WEST ENGLAND}

${ }^{1}$ P Elliott*, ${ }^{2} \mathrm{~N}$ Payne. 'Geography, Lancaster University, Lancaster, UK; ${ }^{2}$ School of Health and Related Research, University of Sheffield, Sheffield, UK 